\title{
Re: Lifestyle-Related Determinants of Obesity Among Omani Children
}

\section{Dear Editor,}

I read with interest the recent study by Al Yazeedi et al. published in the August 2020 issue of SQUMJ. ${ }^{1}$ They studied the correlation between body mass index (BMI) z-scores and lifestyle-related factors, namely nutrition, physical activity, screen time and time spent sleeping among a cohort of 6-10-year-old Omani children. In the study methodology, it is mentioned that BMI were estimated and BMI z-scores was established based on the World Health Organization (WHO) growth charts for children aged 5-19 years. ${ }^{1}$ Accordingly, they found that $17.4 \%$ of the studied children were determined to be overweight or obese. There was no significant correlation between BMI z-scores and nutrition intake, moderate-to-vigorous physical activity time or screen time $(P \geq 0.05)$. However, there was a significant positive correlation between increased time spent sleeping at night and obesity $(P<0.05){ }^{1}$ In addition to the study's limitations that have been mentioned by Al Yazeedi et al., I presume that the following methodological limitations could further influence the above-mentioned study results. ${ }^{1}$ There are many growth trajectory centiles to evaluate growth in children, notably WHO centiles, National Center for Health Statistics (NCHS) centiles and population-specific centiles. Variations do exist in the precision of the different centiles in assessing paediatric growth status. In Oman, the overall prevalence estimates of paediatric underweight, wasting, stunting and overweight were $17.8 \%, 7.4 \%, 10.9 \%$ and $1.3 \%$, respectively, according to NCHS centiles compared to $11.3 \%, 7.6 \%, 13.0 \%$ and $1.9 \%$, respectively, according to WHO centiles. ${ }^{2}$ Moreover, overweight and stunting revealed statistically significantly higher estimates while underweight was statistically significantly lower. ${ }^{2}$ Growth centiles are controlled by various determinants such as age, gender, ethnicity, nutritional status and socioeconomic standard; ${ }^{3}$ population-specific centiles could disclose the growth of children more faithfully compared to other centiles. ${ }^{4}$ Therefore, the generation of Omani population-specific paediatric BMI z-scores, similar to those that have been previously established, is fundamental. ${ }^{5}$ Implementation of the national BMI z-scores could better capture the correlation between obesity and lifestyle-related events among the paediatric population in Oman. Other study limitations, notably the limited sample size and the influence of selecting a narrow age group of 6-10 years in the studied population compared to a wider age group of 5-19 years could substantially impact the study's results. I believe that studying lifestyle-related determinants of obesity using growth charts could imply that obesity is a complex disease and a major paediatric health threat with all its serious sequelae that necessitates directing efforts to implement strategic therapeutic and preventive measures.

\section{Mahmood D. Al-Mendalawi}

Department of Paediatrics, Al-Kindy College of Medicine, University of Baghdad, Baghdad, Iraq

E-mail:mdalmendalawi@yahoo.com

\section{References}

1. Al Yazeedi B, Berry DC, Crandell J, Waly M. Lifestyle-Related Determinants of Obesity Among Omani Children. Sultan Qaboos Univ Med J 2020; 20:e279-86. https://doi.org/10.18295/squmj.2020.20.03.006.

2. Alasfoor D, Mohammed AJ. Implications of the use of the new WHO growth charts on the interpretation of malnutrition and obesity in infants and young children in Oman. East Mediterr Health J 2009; 15:890-8. https://doi.org/10.26719/2009.15.4.890.

3. Ziegler EE, Nelson SE. The WHO growth standards: Strengths and limitations. Curr Opin Clin Nutr Metab Care 2012; 15:298-302. https:// doi.org/10.1097/MCO.0b013e3283511478.

4. Khadilkar V, Khadilkar A. Growth charts: A diagnostic tool. Indian J Endocrinol Metab 2011; 15 Suppl 3:S166-71. https://doi. org/10.4103/2230-8210.84854.

5. Karki S, Päkkilä J, Laitala ML, Ojaniemi M, Anttonen V. National reference centiles of anthropometric indices and BMI cut-off values in a child population in Nepal. Ann Hum Biol 2018; 45:447-52. https://doi.org/10.1080/03014460.2018.1530302. 